Gut, 1985, 26, 724-729

\title{
Acute pancreatitis: a lethal disease of increasing incidence
}

\author{
A P CORFIELD, M J COOPER, AND R C N WILLIAMSON \\ From the University Department of Surgery, Bristol Royal Infirmary, Bristol
}

SUMMARY Between 1968 and 1979650 patients in the Bristol clinical area suffered 737 attacks of acute pancreatitis. Sex distribution was approximately equal and mean age was 60 years. Comparison with the previous decade shows an increase in mean annual incidence of first attacks from 53.8 to 73.0 cases per million population. Case mortality was unchanged at $20 \%$. In no less than $35 \%$ of fatal cases the diagnosis was first made at necropsy. Gall stones were detected in $50 \%$ of first attacks, predominantly in women. The proportion of alcoholics (8\% overall) increased three-fold during the period of the study. In $23 \%$ of cases no aetiological cause was identified. Eighty patients suffered 99 recurrent attacks of acute pancreatitis, with a mortality rate $(12 \%)$ that was not significantly lower than that of the first attack. Neglected gall stones accounted for $51 \%$ of these subsequent attacks.

Wide differences in the incidence of acute pancreatitis have been reported both within and between countries. ${ }^{1-3}$ These fluctuations may reflect environmental differences, for example in alcohol consumption, cholelithiasis or even the hardness of water, ${ }^{2}$ but published series are often difficult to compare. The criteria of diagnosis, the diligence with which case notes and necropsy records have been retrieved, and selection and bias in the local population have either differed or not been mentioned.

Trapnell and Duncan's comprehensive review of acute pancreatitis in the Bristol clinical area from $1950-69^{4}$ reported an average yearly incidence of first attacks of 54 cases per million population, with a mortality rate of 9 per million. We have updated this review to 1979 using data from the same Bristol hospitals, which drain catchment areas in urban Bristol and the surrounding rural parts of Avon, Somerset, and Gloucestershire. We have retained the same diagnostic criteria as Trapnell and Duncan and the same methods of patient retrieval, so far as changes in biochemical assay and hospital activity auditing would allow.

\section{Methods}

\section{PATIENTS}

All recorded cases of acute pancreatitis in the Address for correspondence: Professor R C N Williamson. University Department of Surgery, Bristol Royal Infirmary, Bristol BS2 8HW.

Received for publication 13 August 1984
Bristol area presenting between 1 January 1968 and 31 December 1979 were assessed. An overlap with the earlier review for 1968 and 1969 was necessary, as patients presenting during these years had not been included in the previous statistical analysis. As before, several approaches were used to maximise case retrieval. The hospital activity analysis of the South Western Regional Health Authority was used to identify most cases; all the hospitals serving the Bristol clinical area were included in the computer search. The analysis coding used was $577 \cdot 0$ (acute pancreatitis), and the same code was used to calculate national returns for both incidence and mortality rate. A search of the death certificate records at the Coroner's office revealed a small number of otherwise unrecorded cases. Lastly, during the period under review various clinical trials in acute pancreatitis have been undertaken in Bristol, ${ }^{56}$ and details from these were included. There were no direct referrals from outside the catchment area. A few patients were visitors, but because all of these were in the area at the onset of their attack, they have been included in the survey.

The diagnosis of acute pancreatitis was only accepted if there was a consistent clinical picture supported by biochemical, operative or necropsy evidence of the disease. Two methods of serum amylase assay were used in the area hospitals during the period of survey. For conformity with Trapnell and Duncan ${ }^{4}$ a level of 1000 Somogyi units per 100 $\mathrm{ml}$ (normal <160) was deemed equivalent to 2000 
international units per litre (Phadebas: normal $<300$ ), after several sera had been studied by both methods and a standard curve derived $(r=0.997)$. These values were the minimum necessary for the diagnosis of acute pancreatitis. Obvious pancreatic or peri-pancreatic inflammation was necessary for operative or necropsy diagnosis.

Biliary radiology was usually carried out within six weeks of an attack of pancreatitis, though a few patients were not investigated because of either clinical reticence or patient non-compliance. Alcoholism was defined as a regular intake of 4 pints of beer or a third of a bottle of spirits per day.

The catchment population has been derived from statistics supplied by the South Western Regional Health Authority's medical statistics department: between 1968-79 there were 8745000 person-years at risk. County and local council boundaries have twice undergone extensive reviews during the 12 year study period, necessitating estimation of the population at risk to the nearest thousand persons. Statistical probabilities were calculated using the $\chi^{2}$ test.

\section{Results}

\section{INCIDENCE}

There were 737 documented attacks of acute pancreatitis in 650 patients ( 332 women, 318 men), with a mean age of 60 years (range 3-94 years). Six hundred and thirty eight of these were first attacks of acute pancreatitis, and the remaining 99 were recurrent episodes. Twelve patients in the recurrent group had sustained their first attack either outside the region or before 1968. When related to the general population at risk, the mean annual incidence of a first attack of acute pancreatitis was 73.0 cases per million, with a range of $48 \cdot 9-111 \cdot 2$ cases per million. The incidence increased from a mean of $55 \cdot 1$ for the six years $1968-73$ to a mean of $90 \cdot 5$ for the similar period 1974-79 (Fig. 1). This rise is reflected in an increase in hospital cases from 35 in 1968 to 82 in 1979 (Table 1).

MORTALITY

The outcome was fatal in 125 of the 638 first attacks (mortality rate $19.6 \%$ ), whereas there were only 12 deaths in the recurrent group of $99(12.1 \%$ : $\mathrm{p}<0.08)$. The absolute death rate in relation to the population at risk allows certain comparisons with national statistics, although it must be remembered that diagnostic criteria may vary. Analysis of the Registrar General's figures for the years involved ${ }^{7}$ shows that there were 588418600 person-years at risk in England and Wales and 7323 deaths from acute pancreatitis, giving a mean annual death rate

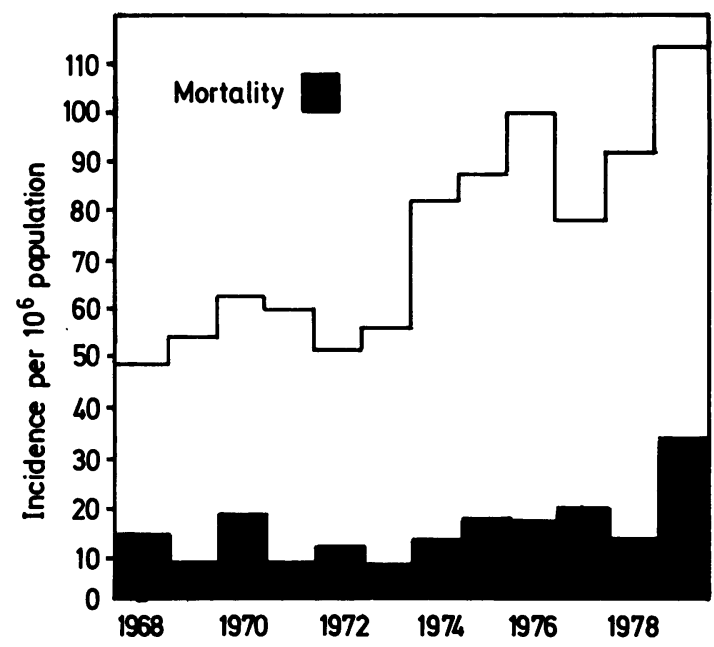

Fig. 1 Incidence and mortality rate of first attack of acute pancreatitis.

of 12.4 per million. These figures include both first and recurrent attacks and contrast with a local mean annual death rate of 15.7 per million (8 745000 person-years at risk and 137 deaths).

\section{DIAGNOSIS}

The diagnosis was established by a consistent clinical picture and serum amylase estimation in 622 cases, at laparotomy in 71 cases and at necropsy alone in 43. The remaining patient had a clinical diagnosis of acute pancreatitis proven at necropsy despite a normal serum amylase. These necropsy cases accounted for $35.2 \%$ of the primary attack fatalities. Four cases were diagnosed at a Coroner's postmortem examination after death at home. The other 39 patients died in hospital with various other premortem diagnoses (Table 2). Thirty five of the laparotomy cases and eight of those diagnosed at necropsy had a serum amylase of less than 1000 Somogyi units per $100 \mathrm{ml}$ on admission. Thus amylase estimation had a $6.5 \%$ false negative rate. Eleven of these 43 cases had amylase levels $>800$

Table 1 Annual incidence of first attacks of acute pancreatitis in Bristol

\begin{tabular}{llrlll}
\hline Year & Cases & Deaths & Year & Cases & Deaths \\
\hline 1968 & 35 & 10 & 1974 & 59 & 9 \\
1969 & 39 & 6 & 1975 & 63 & 12 \\
1970 & 45 & 13 & 1976 & 73 & 11 \\
1971 & 43 & 6 & 1977 & 56 & 13 \\
1972 & 37 & 8 & 1978 & 66 & 8 \\
1973 & 40 & 5 & 1979 & 82 & 24 \\
\hline
\end{tabular}


Table 2 Premortem diagnoses in patients who were diagnosed at postmortem as having acute pancreatitis following death in hospital

Cardio-respiratory failure (death within $24 \mathrm{~h}$ )

Myocardial infarction

Diabetic coma

Hypothermia

Carcinoma of pancreas

Septicaemia - cause unknown

Renal failure

Carcinomatosis

Other ${ }^{*}$

* Including one diagnosis each of hepatic cirrhosis, mesenteric infarction, appendicitis, acute lymphoblastic leukaemia, meningitis, cholecystitis, gastritis, pneumonia, abdominal aortic aneurysm

Somogyi units per $100 \mathrm{ml}$ and a further 12 had levels $>500$ units.

\section{AGE AND SEX}

Patients with a first attack of acute pancreatitis were of mean age 60.0 years (range 3-94 years). Sex distribution was almost equal. Three hundred and twenty seven female patients were of mean age 61.2 years (range 3-92 years), and 311 male patients were of mean age 59.2 years (range 6-94 years). Fatal acute pancreatitis occurred in 102 cases aged 60 or over $(28 \%)$, whereas there were only 23 fatalities below this age $(9 \% ; \mathrm{p}<0.001$; Fig. 2). There were two children under the age of 10 , one of whom had a defect in cell mediated immunity. We have excluded another two children with probable pancreatitis complicating mumps. They had appropriate symptoms and signs, but serum amylase levels only reached 600 and 800 Somogyi units per $100 \mathrm{ml}$.

\section{AETIOLOGY}

Gall stones were detected in $319(50 \%)$ of the patients with first attacks, with a 60:40 female predominance (Table 3). Rather more men with biliary tract disease died than women (17 vs 10\%: $\mathrm{p}<0.08$ ). In 113 patients there was laparotomy or necropsy evidence of common bile duct stones, which were mobile in 99 cases and impacted at the ampulla of Vater in 14 cases. Death was much commoner among patients with impacted stones than those with mobile stones $(71$ vs $11 \%$ : $\mathrm{p}<0 \cdot 001)$. One patient developed acute pancreatitis immediately after an intravenous cholangiogram which showed ductal calculi.

The next largest aetiological group comprised those in whom no obvious factor could be identified despite either radiological investigation, laparotomy or autopsy. This idiopathic group comprised 148 patients $(23 \%)$, with 29 deaths evenly distributed

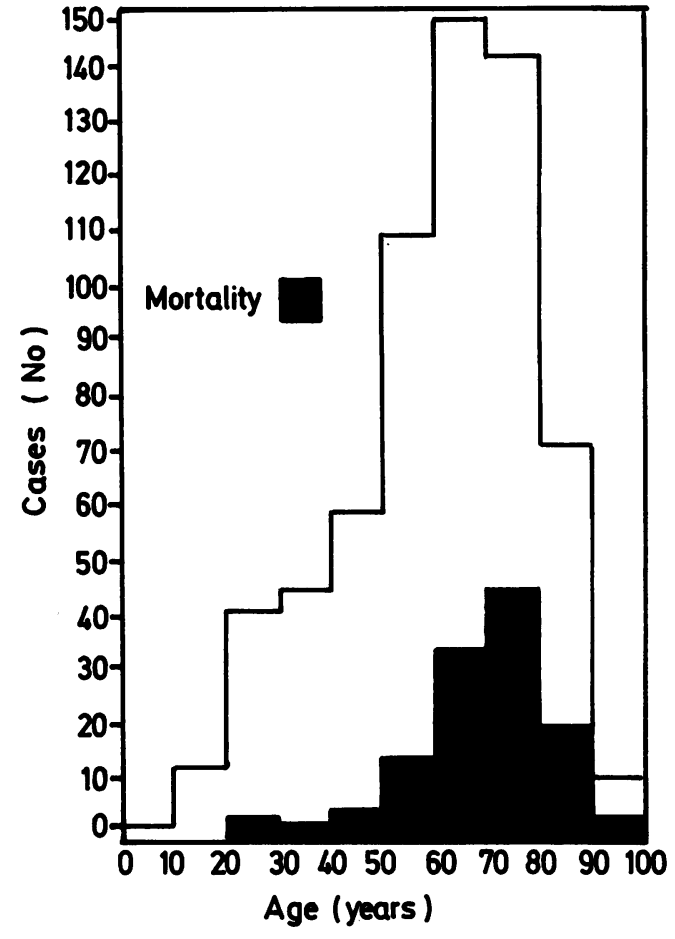

Fig. 2 Age-related incidence and mortality rate of first attack of acute pancreatitis.

between men and women.

Alcoholism caused a total of 51 first attacks, of which $45(88 \%)$ were in men. In the four-year period 1968-71 there were six attacks of acute pancreatitis ascribed to alcohol, between 1972 and 1975 there were 15 , and from 1976 to 1979 there were 30 attacks. These figures show a progressive

Table 3 Aetiological causes in patients with a first attack of acute pancreatitis

\begin{tabular}{lrrrrr}
\hline & \multicolumn{2}{c}{ Cases } & & & \multicolumn{2}{c}{ Deaths } \\
\cline { 2 - 3 } \cline { 5 - 6 } Aetiology & $M$ & \multicolumn{1}{c}{$F$} & & $M$ & \multicolumn{1}{c}{$F$} \\
\hline Gall stones & 130 & 189 & & 22 & 19 \\
Alcohol & 45 & 6 & 7 & 0 \\
Idiopathic & 72 & 76 & & 15 & 14 \\
Postoperative & 10 & 10 & & 5 & 6 \\
Neoplasia & 7 & 4 & & 5 & 4 \\
Other* & 25 & 19 & & 12 & 7 \\
Unknown & 22 & 23 & & 4 & 5 \\
Total & 311 & 327 & 70 & 55 \\
\hline
\end{tabular}

* Including: hyperlipidaemia (6), blunt abdominal trauma (3), hyperparathyroidism (2), mumps (1), isolated dorsal pancreas (1), septicaemia (1) and cytomegalovirus infection (1) 
increase in alcohol-related acute pancreatitis from $3.7 \%$ of attacks to $10.8 \%$.

Twenty patients suffered their first attack of acute pancreatitis after a surgical operation or investigation. Relevant procedures included transduodenal sphincteroplasty $(n=4)$, vagotomy and pyloroplasty (three), gastrectomy (three), right hemicolectomy (two), translumbar aortography (two), and one each for endoscopic retrograde cholangiopancreatography (ERCP), aspiration biopsy of the pancreas, cholecystectomy (alone), bilateral adrenalectomy, division of adhesions and cardiopulmonary bypass. Postoperative pancreatitis was fatal in 11 of 20 cases $(55 \%)$, including all three gastrectomies and three of the four sphincteroplasties.

Acute pancreatitis was associated with malignant neoplasia in 11 patients, usually as a terminal event. There were three pancreatic carcinomas, one hepatoma, one gall bladder carcinoma, one leukaemia and five peripancreatic secondaries. Although 24 patients (nine deaths) were taking corticosteroids, 10 of these had other aetiological factors. Likewise 21 patients (nine deaths) had diabetes mellitus but 10 had other factors. Hypothermia $\left(<34^{\circ} \mathrm{C}\right)$ was recorded on admission in eight patients, half of whom died. Four patients developed acute pancreatitis during pregnancy or in the puerperium; three had biliary disease and one was an alcoholic.

\section{RECURRENT PANCREATITIS}

Recurrent attacks of acute pancreatitis occurred in 68 of the $513(13 \%)$ patients who survived a first attack during the period under review. Another 12 patients had a previous attack of acute pancreatitis conforming to our diagnostic criteria, either outside the Bristol area or before 1968. The mean age, at their first attack, of patients with recurrent disease was 53.5 years (range 17-88 years). The total of 80 patients (44 women, 36 men) suffered 99 recurrent attacks; 13 had three attacks and another three had four attacks. In all, there were 12 deaths in this group $(12 \cdot 1 \%$, Table 4$)$.

Most recurrent attacks $(51 \%)$ were in patients with cholelithiasis, who were either awaiting cholecystectomy or in whom operation had been declined by patient or surgeon. Eighteen patients (two deaths) had stones in the common bile duct. In only one of these was a stone impacted at the ampulla of Vater, and this patient made an uneventful postoperative recovery.

Recurrent attacks were documented in 12 of the 38 male alcoholics who survived a first attack during the period of review. One man had two further attacks, and one had had the first attack before 1968. These alcoholic patients had no evidence of chronic pancreatitis. Lastly, three octogenarian
Table 4 Aetiological causes in patients with recurrent acute pancreatitis

\begin{tabular}{lrrrr}
\hline & \multicolumn{3}{c}{ Recurrent attacks $($ no $)$} & \\
\cline { 2 - 4 } Aetiology & 1 & 2 & 3 & Deaths \\
\hline Gall stones & 34 & 5 & 2 & 4 \\
Alcohol & 12 & 1 & & \\
Idiopathic & 14 & 6 & 1 & 5 \\
$\begin{array}{l}\text { Steroids } \\
\text { Hyperlipidaemia }\end{array}$ & 1 & & & 1 \\
$\begin{array}{l}\text { Dorsal pancreas } \\
\text { Unknown }\end{array}$ & 1 & 1 & & \\
Total & 2 & & & 2 \\
\hline
\end{tabular}

women died from recurrent pancreatitis; one was on steroid therapy and all three had pre-existing cardiorespiratory disease.

\section{Discussion}

INCIDENCE AND MORTALITY

The mean annual incidence of acute pancreatitis in Britain has variously been reported between $48^{2}$ and $238^{3}$ cases per million population. For valid geographical comparisons, all cases of the disease occurring in each area must be included, using the same diagnostic criteria. Prospective surveys have the drawback of excluding cases not presenting to hospital clinicians. It is well recognised that different areas have different patterns of disease, so that extrapolation of local figures to obtain national statistics is fallible.

As acute pancreatitis is seldom treated by early operation, clinical suspicion of the disease is generally confirmed by hyperamylasaemia. The precise concentration of serum amylase that can be taken as diagnostic is controversial, however. The values used in the present study were chosen to permit direct comparison with the previous Bristol survey. ${ }^{4}$ They represent between six to seven times the upper level of normal in our laboratories. Similar rises are rarely obtained in other conditions, ${ }^{89}$ most of which demand prompt laparotomy. By contrast, the false negative rate of $6.5 \%$ suggests that we have overlooked some patients with mild oedematous pancreatitis.

Compared with Trapnell and Duncan's previous report, ${ }^{4}$ our survey shows a $26 \%$ increase in acute pancreatitis in Bristol. Local interest in the disease because of clinical trials seems unlikely to explain this increase, because the proportion of mild cases has remained unchanged. The incidence of acute pancreatitis in relation to age, sex and predisposing factors agrees with other published British series; ${ }^{2} 41011$ more women had underlying chole- 
lithiasis and more men were alcoholics.

Many reports have suggested that the mortality rate of acute pancreatitis is decreasing, ${ }^{5612} 13$ owing perhaps to earlier diagnosis and treatment following heightened awareness of the disease; but being prospective in nature, these studies have overlooked cases in which pancreatitis was unsuspected before death. ${ }^{14}$ In our survey, a little more than one third of the patients who died were not diagnosed as having acute pancreatitis premortem. If these patients are excluded, a mortality rate of $13.6 \%$ is obtained. Nevertheless, it is salutary to note that the true rate of $19.6 \%$, which varied but little from year to year, has not improved since $1961-67,{ }^{4}$ despite a number of recent therapeutic trials and advances in intensive therapy.

\section{AETIOLOGY}

The proportion of pancreatitis patients with gall stones $(50 \%)$ has not altered over a 30 year period in Bristol. ${ }^{4}$ The high incidence of common bile duct stones $(35 \%)$ supports the hypothesis that the causative mechanism in gall stone pancreatitis is the passage of a calculus through the common channel formed by pancreatic and common bile ducts, with consequent reflux of bile or duodenal juice into the pancreatic duct. ${ }^{15-17}$ Impaction of a stone at the ampulla of Vater in $56 \%$ of necropsies showing gall stone pancreatitis lends credence to this theory and suggests that early operative intervention should be considered if this condition is suspected.

Alcoholism appears to be a much commoner cause of acute pancreatitis elsewhere in Britain and abroad, ${ }^{9}{ }^{18-21}$ although it has steadily increased over the last 30 years in Bristol. ${ }^{4}$ Our findings confirm that postoperative pancreatitis usually follows upper abdominal surgery and is a dangerous disease with a $55 \%$ chance of death. ${ }^{22}$ The aetiological relevance of steroids is disputed $;{ }^{4} 23$ nearly half our patients on steroids had other predisposing factors. Our data suggest that diabetes is no more prevalent in acute pancreatitis than in the general population despite a previous report to the contrary. ${ }^{20}$ Pre-existing diabetes, however, does increase the risk of death from 19 to $43 \%$. Concomitant hypothermia is even more lethal, though it could either precede or follow the attack of acute pancreatitis. ${ }^{24}$

Other aetiological associations of acute pancreatitis, such as hyperlipidaemia and hyperparathyroidism, make up an expected small percentage of cases. ${ }^{14} 1820$ Like others, ${ }^{425}$ we have found no convincing evidence that pregnancy is a relevant factor. There was only one case of acute pancreatitis after ERCP, though the reported incidence is $1-2 \%{ }^{26}$ Because over 900 ERCP examinations were performed during the study period, several mild attacks of pancreatitis were probably missed.

\section{RECURRENT ATTACKS}

Though the second and subsequent attacks of acute pancreatitis seem to be less dangerous than the first, this difference is not as great as originally suggested. ${ }^{4}$ Delayed cholecystectomy is still the major factor in recurrent pancreatitis, accounting for half the cases; subsequent attacks of gall stone pancreatitis are almost as lethal as the first attack. Likewise, in idiopathic pancreatitis the mortality rate of subsequent attacks is similar to that of the initial attack. After one attack of acute alcoholic pancreatitis some patients develop a chronic relapsing disease with functional impairment; others suffer recurrent acute attacks, though these did not prove fatal in our experience.

\section{References}

1 O'Sullivan JN, Nobrega FT, Morlock CG, Brown AL, Bartholomew LG. Acute and chronic pancreatitis in Rochester, Minnesota, 1940-1969. Gastroenterology 1972; 62: 373-9.

2 Bourke JB, Giggs JA, Ebdon DS. Variations in the incidence and the spatial distribution of patients with primary acute pancreatitis in Nottingham 1969-76. Gut 1979; 20: 366-71.

3 Graham DF. Incidence and mortality of acute pancreatitis. Br Med J 1977; 2: 1603.

4 Trapnell JE, Duncan EHL. Patterns of incidence in acute pancreatitis. Br Med J 1975; 2: 179-83.

5 M.R.C. Working Party. Death from acute pancreatitis: multicentre trial of glucagon and aprotinin. Lancet 1977; 2: 632-5.

6 Cooper MJ, Williamson RCN, Pollock AV. The role of peritoneal lavage in the prediction and treatment of severe acute pancreatitis. Ann R Coll Surg Engl 1982; 64: 422-7.

7 Registrar General's statistical review of England and Wales. Part I: medical tables. London: HMSO, 19691980.

8 De Jode LRJ. Acute pancreatitis. In: Taylor S, ed. Recent advances in surgery 9. London: Churchill Livingstone, 1977: 83-111.

9 Ranson JHC. Acute pancreatitis - where are we? Surg Clin N Am 1981; 61: 55-70.

10 Imrie CW, Whyte AS. A prospective study of acute pancreatitis. Br J Surg 1975; 62: 490-4.

11 Read G, Braganza JM, Howat HT. Pancreatitis - a retrospective study. Gut 1976; 17: 945-52.

12 Imrie $\mathrm{CW}$. Incidence and mortality of acute pancreatitis. Br Med J 1977; 2: 1350.

13 McMahon MJ. Incidence and mortality of acute pancreatitis. Br Med J 1977; 2: 1350.

14 Bourke JB. Incidence and mortality of acute pancreatitis. Br Med J 1977; 2: 1668-9. 
15 Opie EL. The aetiology of acute haemorrhagic pancreatitis. Bull Johns Hopkins Hosp 1901; 12: 182-8.

16 Acosta JM, Pellegrini CA, Skinner DB. Aetiology and pathogenesis of acute biliary pancreatitis: Surgery 1980; 88: 118-25.

17 McCutcheon AD, Race D. Experimental pancreatitis: a possible aetiology of postoperative pancreatitis. Ann Surg 1962; 155: 523-31.

18 Ranson JH, Spencer FC. The role of peritoneal lavage in severe acute pancreatitis. Ann Surg 1978; 187: 565-75.

19 Marks IN, Bank S. The aetiology, clinical features and diagnosis of pancreatitis in the South Western Cape: a review of 243 cases. $S$ Afr Med J 1963; 37: 1039-53.

20 Jacobs ML, Daggett WM, Civetta JM et al. Acute pancreatitis: analysis of factors influencing survival.
Ann Surg 1977; 185: 43-51.

21 Gillespie WJ. Observations on acute pancreatitis. A retrospective clinical study. Br J Surg 1973; 60: 63-5.

22 White TT, Morgan A, Hopton D. Postoperative pancreatitis: a study of 70 cases. Am J Surg 1970; 120: 132-7.

23 Steinberg WM, Lewis JH. Steroid-induced pancreatitis: does it really exist? Gastroenterology 1981; 81: 799-808.

24 Savides EP, Hoffbrand BI. Hypothermia, thrombosis and acute pancreatitis. $\mathrm{Br} \mathrm{Med} J$ 1974; 1: 614.

25 McKay AJ, O'Neill J, Imrie CW. Pancreatitis, pregnancy and gallstones. Br J Obstet Gynaecol 1980; 87: 47-50.

26 Cotton PB. Progress report: ERCP. Gut 1977; 18: $316-41$. 